\title{
Simulation and Optimization Based Flexible Job Scheduling of Powder Coating Lines
}

\author{
Heike Wilson $^{1}$ and Reik Zettl ${ }^{2}$ \\ ${ }^{1,2}$ DUALIS GmbH IT Solution \\ hwilson@dualis-it.de \\ www. dualis-it.de
}

\begin{abstract}
The Paper will present a new approach to use simulation methods combined with intelligent optimization algorithms in an operative scheduling and monitoring application for powder coating lines. Powder Coating lines are built usually as a closed loop "power and free" chain system that transports the products on carriers from a starting point through the different production stages. Due to the closed loop, efficiency and the overall output of the system is highly dependent on the sequence of the production orders entering the line. Inefficient sequences can cause deadlocks, significant decrease of the production output or even scrap production. The presented application architecture is based on a 3D simulation platform working together with a closed loop heuristic sequence scheduling algorithm. The paper will also introduce the system architecture design with full integration to an enterprise IT structure comprising ERP, real time PLC communication and a Warehouse Management System.
\end{abstract}

Keywords: Material flow simulation, Advanced planning and scheduling, optimization, powder coating lines, ERP, PLC, shop-floor control, order sequencing, production order mix, delivery dates, multi-criteria optimization, 3D simulation.

\section{Introduction}

Globalisation and the associated pressure on costs has increased considerably during the last decades and most production companies, especially small and medium size enterprises "SMEs", are forced to continually adapt, most often through better production flexibility and efficiency. Surface treatment and coating production usually supplies products to internal finishing and assembly lines or to external customers, but always "just-in-time" or "just-in-sequence" where the throughput-time is as short as possible. Quite often production order information arrives at very short notice to the coating process leaving little time to make a decision, let alone an optimal one, about the order sequence. Ideally the decision must be based on various parameters such as status of current production orders, requested delivery dates, equipment and buffer utilization and order properties like sizes and colours and so on. This complex information increases the pressure on optimized planning combined with a high product quality and processes stability. Most companies still rely on manual scheduling 
and have to learn from painful experiences of lost earnings and efficiency before thinking more about lean manufacturing processes, integrated with flexible and affordable IT-Systems. Manually based solutions limit the information flow between the ERP level and shop floor production management, placing an increased burden on the coordination between departments.

In contrast, large manufacturing enterprises have been utilising technologies based on industrial simulation toolkits and mathematical based optimisation algorithms to support shop floor production planning already for some time. In the presented solution based on successful implemented project examples, available simulation technologies were combined with automatic scheduling in one decision support system that delivers required information to the planning department very fast and always based on the current production system status. The available 3D model of the powder coating line was enriched with additional operation logics, linked to the PLC and the ERP-system, and combined with fast heuristic scheduling algorithms in one integrated IT platform. The results of the scheduling is used to constantly control and optimize the material flow inside the powder coating line by delivering optimal decisions for each production stage.

The advantage of such a solution where 3D simulation models can be reused for supporting tasks like scheduling and line control is high for an SME. However few industrial case studies exist where Virtual Factory models initially used for layout proposals or a simulation study have been re-used for production scheduling, monitoring and control.

\section{Project Overview}

\subsection{Project Partners}

Rippert Anlagentechnik GmbH \& Co.KG is the system supplier of both implemented powder coating systems. The are located in Herzebrock-Clarholz, where the mid-size company successfully holds its own in the market with three divisions covering coating plants, filter systems and industrial fans. This system supplier has been developing, producing and installing state-of-the-art, energy-saving plants for industrial customers all over the world for the last 45 years.

As a long term application user of the 3D Simulation toolkit (powered by the Visual Components Oy) Rippert was also very interested in the idea to enlarge their own plant visualization system with simulation and optimization based technologies. The main idea from their side was to offer the customer a new integrated solution providing four additional functionalities besides the visualization - 3D simulation, line monitoring and control and above all intelligent optimization based order scheduling.

The second project partner the mid-size company Louis Schierholz GmbH provided the Power and Free closed loop system inside the powder coating process. Schierholz Power \& Free offers optimum flexibility by using a revolving chain on a closed loop, with unit load conveyors shifting loads on a running gear connected to a rail. The running gear and chain are linked via an interlocking catch system. Running gears can change chains at transfer points, and material flows can be separated or 
combined. Stoppers keep the running gears separate from the conveying chain, and help create storage areas, feeding, removal and operation points. One of the main tasks in the project was to connect the 3D simulation model with the Schierholz PLC in order to get the online information for the gears in the operating system to control the process flow for keeping optimal production sequencing.

DUALIS GmbH IT Solution the third implementation partner was responsible for the software development and integration of the IT-Platform at the customer's plants. The company is located in Dresden, Saxony, and has been providing simulation consultancy and software solutions as well as optimization based scheduling applications for over 20 years now. Founded in 1990 by Professor Wilfried Krug, DUALIS now has over 200 worldwide customers who have been able to improve and optimise their production processes with tools based on specialised mathematical algorithms for optimisation. DUALIS mathematicians and developers work together to improve techniques for solving real world optimisation and scheduling problems in the Automotive, Packaging, Automation Assembly and Logistics industries. The company's latest software development is an optimisation based short term scheduling architecture for small and medium sized companies in the production planning area. The architecture has a planner and constraint solver algorithm optimising for several and often conflicting optimisation goals based on a production system with finite resources and capacity. Expanding their applications with shoop-floor and PLC connectivity is a logical step to offer complete solutions based on simulation and optimization and to meet actual customer requirements.

\subsection{Application Description}

In today's manufacturing environment simulation techniques are used to reduce risk mainly in the planning phase for "green field" production plants or the reorganisation of an existing facility. Both 2D and 3D industrial software packages are available for these types of tasks and the derived models are often very detailed and rich in process information. In many cases logistics functions and physical detail is so close to the finally implemented system an opportunity exists to reuse the models to support the end-user's daily processes, such as production scheduling.

Driven by the challenge to get the highest possible efficiency from their powder coating systems, Rippert uses 3D Simulation to optimize the Power and Free system, buffer areas and system parameters during layout design in an early sales phase. With pre-defined product mixes, simulation runs are used to optimize the utilization of the processes and show the client the virtual system with product data as realistically as possible in a "virtual plant". After the planning phase is finished and product parameters like output per shift are agreed and fixed, expectations for real line operation need to be met. The Rippert application used existing simulation models for operative short term scheduling and line monitoring. An approach that can be of particular interest to other line builders and modular equipment suppliers who are already working with complete machine model libraries and working 3D simulation layout models for presales. These types of companies, would be able to extend the use of the presales models and provide a scheduling tool as an option for every turn-key production system 
delivered: to help their customers get the best out of the production system. This applies to most operators that need to schedule, monitor and optimize daily production and react when operational parameters change. In particular, manual changes to production sequencing in a closed loop chain driven system is very hard to successfully accomplish. Sequence scheduling before production start is very important. The Rippert application includes manual scheduling as well as heuristic optimization which can be adapted and customized if needed. The operator is able to define the product route and process times either manually or by importing the actual order pool from the MES (Manufacturing Execution System) and apply only changes. Based on the actual system load the generated sequences can be simulated and evaluated by the operator using defined KPIs. The optimal schedule is created using the integrated optimization engine with different optimization goals, production constraints and parameter settings. Once the sequence is determined it can be uploaded to the M.E.S to load new production order information to the real system. But uploading the optimal sequence is only one side. The many decisions points in the system need ot also also controlled in order to apply the optimal schedule to the system. Especially for the closed loop system minimal changes in the product sequence can cause trouble setup requests at the coating cabins or even deadlocks at critical for example oven processes or buffer areas. Therefor the final integration also links to the sensors and PLC controls in order to manage the decision at switches, feeding, operation and/or removel points as an essential task to also fulfil the optimal schedule.

\subsection{Integration Concept}

Currently SME companies are managing their production facilities using different IT applications. In most cases the applications have grown organically based on specific user needs. As a result, many of the IT sub-systems within one company have evolved with duplicate functionality. The end result is a confusing IT landscape where it is unclear which sub-system can and should deliver the required services, or where new functionality should be developed.

Missing from the SME IT portfolio is an integrating architecture where the required functionality is available through one common interface. Efforts have been focused on ERP (Enterprise Resource Planning) systems but there is little connection to the shop floor, especially in the areas of planning and optimisation. In the last couple of years a clear need has emerged for the research and development of improved ERP support tools for production.

These applications are commonly referred to in larger enterprises as APS (Advanced Planning and Scheduling) tools. To support an integrated solution for the SME with full Product Life Cycle Management the APS application must interface on the ERP side to a master data and to the shop-floor PLC on the other side.

The project is based on the most common vertical integration within the IT infrastructure between ERP, APS and MES/PLC applications as depicted in the following diagram (e.g., Fig. 1) 


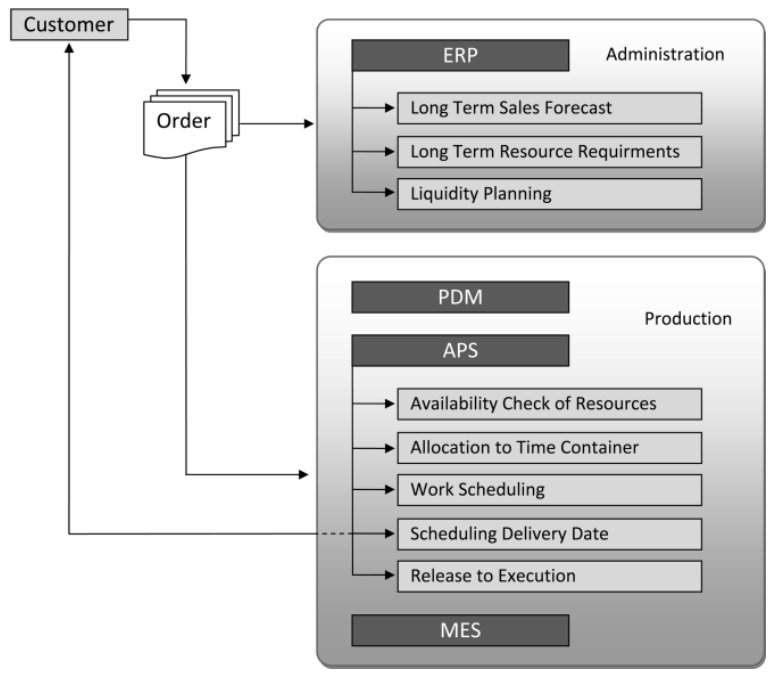

Fig. 1. Vertical Integration of Administration and Production systems

The main administration and order data is stored in the ERP-system. The pilot project ERP-systems were using common databases such as MS SQL and Oracle. In order to stay platform neutral the application connects this Administration level with the production MES level using stored procedure links implemented in C\#. The goal was to implement a generic interface in order to reuse the connection strings in further projects with different ERP-systems, databases and PLC-applications.. More implementation work was required at the APS-level. The 3D simulation models used for system design and optimization during pre-sales and engineering had to be supplemented with PLC information representing the real shop-floor system. To monitor using a $3 \mathrm{D}$ model requires a unique and realistic representation of each Power and Free section and production process stage.

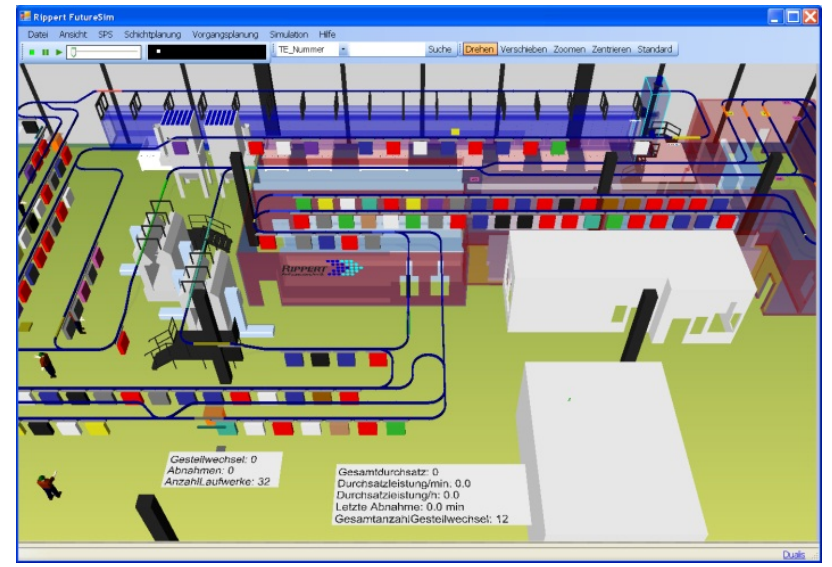

Fig. 2. Operating 3D model of an Rippert powder coating system 
Every section of the Power and Free system is modelled with start and end sensors to register the information of the entering and leaving gears. In order to visualize the correct gears and information about the transported products in each sector the sensors need to be modelled with real-time connectivity to the PLC/OPC-application data. In each section the actual number of possible gear buffers are implemented. Each buffer place has a unique address managed by the PLC-server. With the provided address the APS-application can check if the sections are loaded and carry out the real time optimization process based on this information. For each optimization run the actual gear load in each system will be read from the PLC using this adress. Product information like type, colour and other important specifications are available from the PLC data as an input to the optimization. Based on this information the scheduling heuristics creates now new sequences in different areas based on the optimization goals set. The project experience shows that the setup time optimization at the powder cabins and promise to deliver at the final assembly are most important. The main goal is to schedule long colour batches with minimized cleaning and setup times between different colours. Lowering the down times at the colour cabins increases the system efficiency and overall product output significantly. Besides setup optimization in more complex powder coating lines the decision points at intersections and crossings become more important. The same applies to product mixes with repeating product routes. Since the decision of which gear will be released for instance from buffer areas will have an impact later on at following productions stages. Therefore the actual status must be updated almost online from the PLC, adjustable to the user requirements.

\section{Conclusions}

The benefits for the planner in comparison to available short term planning based options, that do not use simulation and optimization is the ability to quickly pre-test a calculated optimal schedule, and allow visual feedback to the planner about the actual system status combined with planning results. Further more using the PLC linkage the schedule will be online updates and adapted to the actual situation at any time. System deadlock and unnecessary colour setups are minimized. Both user plants increased the number of gears going through powder coating per shift by about $15 \%$. The next challenge will be to not to look only at the coating line as single optimum but also include all pre-successors and successor stages into the optimization model in order to achieve a total optimum for the plant.

General benefits from using simulation and optimization as well as the operative scheduling benefit can be summarised with:

- Modelling of complex systems and networks for better understanding

- Fast and flexible adaptation to changing production system environment

- optimized production schedules

- Test "what-if" scenarios on the virtual production system without any real term consequences such as production loss and interruptions.

- Fast scenario testing and future prediction capability for planning purposes 
- Better understanding of the production system

- Cost effective validation of new ideas or strategies

- Faster analyses and results

- optimized production schedules with cost minimization and more overall system efficiency 